الأكاءات المتعددة وقوة الأنا لاى الطلاب

الأكاعات المتعددة وقوة الأنا لاى الطلاب الجامعة 
د/ سامية سمير شحاتة

مقدمة ومثكلة الدراسة :

(Yr.: 1995

$(117-110: 1997$

$(r r: r m)$ 
الأكاءات المتعددة وقوة الأنا لاى الطلاب
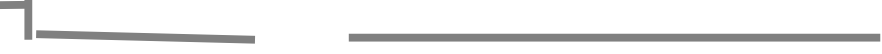
د/ سامية سمير شحاتة

(0) : 19Ar

(V7: 1974

Na: Yor

(10r: 199V

(1) $199 V$ 
الأكاءات المتعددة وقوة الأنا لدى الطلاب

1990: 518)

Koening ; April R.Trees et al. ,2010) 
د/ سامية سمير شحاتة

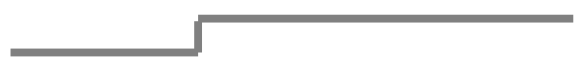


الأكاءات المتعددة وقوة الأنا لاى الطلاب
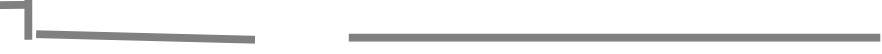
ع - تأمل الباحثة أن تثجع الدراسة طلبة الدراسات العليا، والمهتمين بالبحث

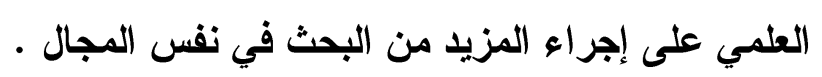

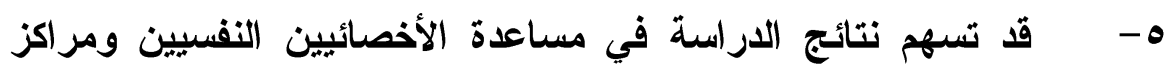

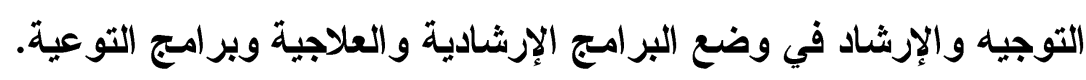

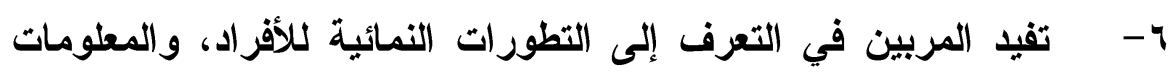
العصبية البيولوجية التي يتعلمونها عن الدماغ، وأن الأكاء متعدد وليس موحدا.

Multiple Intelligence 
الذكاءات المتعددة وقوة الأنا لاى الطلاب

(Nelson,1998 :9).

(Tr: 
د/ سامية سمير شحاتة

(rq: rol

1 . 
الأكاءات المتعددة وقوة الأنا لاى الطلاب

(10: r r r

$.00:$ Y I E

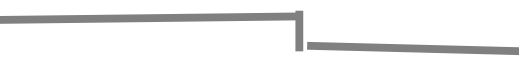


د/ سمية سمير شحاتة

(Gardner \& Hatch , 1989 :5 \& Harry 1992 : 29 ,)

\& Hatch , 1989 :6)

(rvo: 199V

(T: Y Y T

IT 
الأكاءات المتعددة وقوة الأنا لدى الطلاب

Jody Koening ( $r$ : Y Y צ

$\left(V \leq: r_{m}\right.$

Checkley ,1997 : 8-13) 
د/ سامية سمير شحاتة

(Checkley ,1997 : 8-13)

:

: 
الذكاءات المتعددة وقوة الأنا لاى الطلاب

\section{Ego Strength}

:

( $14: 1991$

(749: 1994

(Y97: 1998 
د/ سامية سمير شحاتة

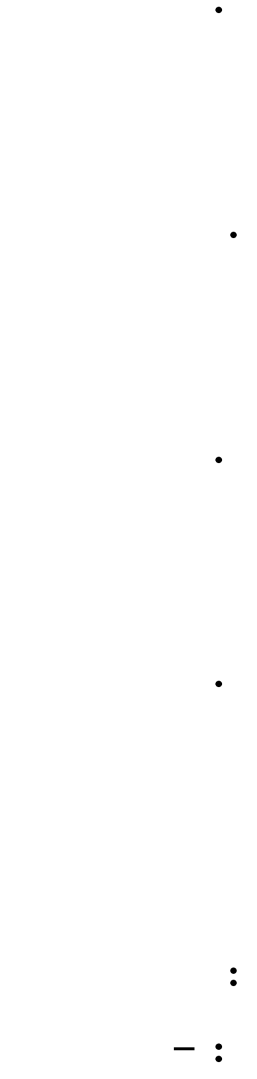

(Ar : 199V.

( I V.: 1987 
الأكاءات المتعددة وقوة الأنا لاى الطلاب

(ro: 199

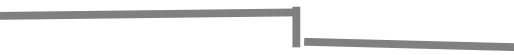


د/ سامية سمير شحاتة

1- النظرية التحليلية:

11 
الذكاءات المتعددة وقوة الأنا لاى الطلاب

(r)o: 1991

$(10-1 .: 1991$

$(191-110: 19 \vee 9$

r- نظرية عوامل الثخصية لريموند كاتل:. 
د/ سامية سمير شحاتة

(VE: Yur

$$
(v .-79: 1997
$$

.(r99- rAY: 1991

تعقبي:

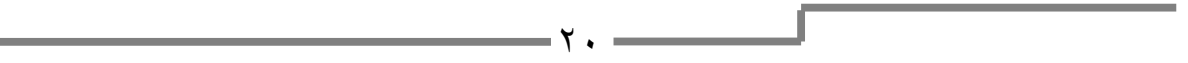


الأكاءات المتعددة وقوة الأنا لاى الطلاب

: 
د/ سامية سمير شحاتة

$(110-11 \leq: 1994$

(rT: Yus

(1)人: 1994

:

:

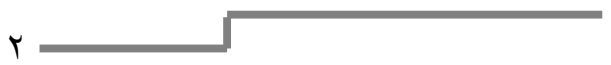


الأكاءات المتعددة وقوة الأنا لاى الطلاب

$(199 v)$

(199V)

(rm) Snder

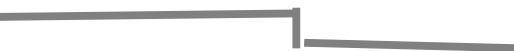


د/ سامية سمير شحاتة

(Y.")

$(r+1)$

$r \varepsilon$ 
الأكاءات المتعددة وقوة الأنا لاى الطلاب

Markstrom \& Kalmanir(2001 دراسة ماركستروم وكالمنير)

(Y«) Furnham , Akande 
د/ سامية سمير شحاتة

markstrom et al.,(2007)

$\cdot($

: ry 
الأكاءات المتعددة وقوة الأنا لاى الطلاب

Psychosocial Inventory of القائمة النفس اجتماعية لقوة الأنا Ego Strength (PIES)

Markstrom, C., Sabino, V., Turner, B., \& Berman, (1 १৭४)

R. (1997). 
الوصف الأساسى للمقياس

Psychosocial Inventory of Ego Strengths (PIES)

(Markstrom \& Marshall, 2007; Markstrom, Sabino, Turner, \& Berman, 1997)

(Jody Koening ; . 2010)

April R.Trees et al 
الأكاءات المتعددة وقوة الأنا لاى الطلاب

(Jody Koening ; April R.Trees et al. ,2010) .- Restraint

Koening ; April R.Trees et al. ,2010)

• وتعنى قدرة الفرد على تحديد معنى وجوده و أهدافه فى الحياة ووضوح خططه لتحقيق هذه الأهداف . (Jody Koening ; April R.Trees et al. 2010)

(Jody Koening ; April R.Trees et al. ,2010) • 
April R.Trees et al. ,2010)

\begin{tabular}{|c|c|c|c|}
\hline أرقام البنود العكسية & أرقام البنود الايجابية & البعد & p \\
\hline$\Gamma \wedge, r \leq 6 r T, I \varepsilon$ & $7.60 V_{6} V_{6} 1$ & love الحب & 1 \\
\hline $0 \leqslant$ 6 M ، YV ، & $7 r 6 r r 6196 r$ & الإر ادة Will & $r$ \\
\hline $7 \varepsilon 6716 r 9611$ & $096 r 0,1 \Gamma, r$ & الكفاءة competence & $r$ \\
\hline$\varepsilon \wedge$ ، $\leqslant 161 \cdot 67$ & 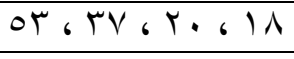 & التفانى fidelity & $\varepsilon$ \\
\hline $0.6 \leqslant r_{6} Y_{6}, I T$ & $07 ، \leqslant 96 r 160$ & الحكمة wisdom & 0 \\
\hline $0 \wedge$, $\leqslant \leqslant 6 \leqslant Y$, YY & rq, Mr. 1061 & الأمل Hope & 7 \\
\hline 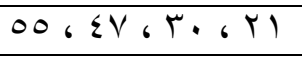 & Or 6 $\leqslant 7$, IV. 9 & care الر عاية & $\mathrm{V}$ \\
\hline 0165.610617 & $T r, \leqslant 0, r \Lambda, r \leq$ & purpose الهدف & $\wedge$ \\
\hline
\end{tabular}


الأكاءات المتعددة وقوة الأنا لاى الطلاب

\begin{tabular}{|c|c|c|c|c|c|}
\hline ألفا كرونباخ & أعادة الاختبار & الثبات & ألفا كرونباخ & أعادة الاختبار & \\
\hline.$V \varepsilon$ &.$v 9$ & الأمل &.$v 9$ &.$V r$ & الحب \\
\hline.$V r$ &.$v V$ & الرعاية &.$\vee \wedge$ &.$v 0$ & الإر ادة \\
\hline.$V Y$ &.$V 4$ & الهـف &.$v V$ & $\cdot v_{1}$ & الكفاءة \\
\hline \multirow[t]{2}{*}{$\cdot, v_{1}$} & $\cdot, V Y$ & المجموع الكلى & 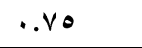 & 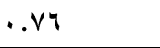 & التفانى \\
\hline & & &.$V 4$ &.$\vee \wedge$ & الحكمة \\
\hline
\end{tabular}

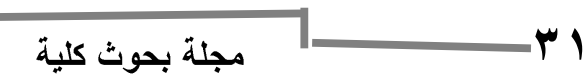

الآداب 


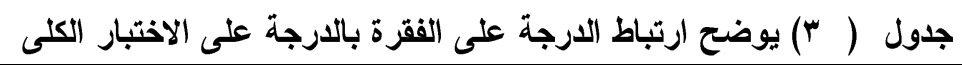

\begin{tabular}{|c|c|c|c|c|c|c|c|}
\hline معامل الارتباط & رقم البند & J & م & J & p & معامل الارتباط & رقم البند \\
\hline., 791 & $\varepsilon 9$ & . & $r r$ & $\cdot, 1 \wedge 9$ & iv &., 79. & 1 \\
\hline$\cdot, v \cdot 1$ & o. &., 791 & $\Gamma \varepsilon$ & $\cdot, \vee \ldots$ & 11 &.,$Y \wedge \wedge$ & $r$ \\
\hline., 794 & 01 & .,794 & ro &., 791 & 19 &.,$\uparrow \wedge \vee$ & $r$ \\
\hline., $7 \wedge \wedge$ & or &., $79 \mathrm{~V}$ & ry &., $79 \mathrm{~V}$ & $r$. &., $7 \wedge \wedge$ & $\varepsilon$ \\
\hline ., TqY & or & .,794 & $r v$ &., 797 & rI & $\cdot, v \cdot r$ & 0 \\
\hline., 799 & 0 \& & $\cdot, 7 \wedge 9$ & ru &., 790 & $r r$ &., $79 \leq$ & 7 \\
\hline., 794 & 00 & .,79r & rq &., 790 & $r r$ & . & v \\
\hline., $7 \wedge \wedge$ & 07 &., $79 \leq$ & $\varepsilon$. &., $79 \mathrm{~V}$ & $r \varepsilon$ &., 794 & $\wedge$ \\
\hline., 791 & $\Delta V$ &., $79 \mathrm{~V}$ & $\{1$ &., 799 & ro &., $79 \vee$ & 9 \\
\hline$\cdot, v \ldots$ & 01 &., 791 & $\varepsilon r$ &., 79. & $r y$ &., 797 & 1. \\
\hline$\cdot, \uparrow \wedge \wedge$ & 09 &., 79\{ & $\varepsilon r$ &., 799 & $r V$ &., 790 & 11 \\
\hline., 79. & 7. & $\cdot, v, 0$ & $\varepsilon \varepsilon$ & $\cdot, 799$ & $r \wedge$ & $\cdot, 791$ & ir \\
\hline$\cdot, 794$ & 71 & $\cdot, 794$ & $\{0$ & .,794 & ra & $\cdot, \vee \ldots$ & ir \\
\hline., 791 & Tr &., 790 & $\{4$ & . & $r$. & $\cdot, \vee \cdots$ & $1 \varepsilon$ \\
\hline., $79 \leq$ & Tr &., 790 & $\varepsilon V$ & & rI & $\cdot, \vee \cdots$ & 10 \\
\hline., 790 & $T \varepsilon$ &., 797 & $\{\wedge$ &., 791 & rr &., 791 & 17 \\
\hline
\end{tabular}

\section{Validity}

Content Validity 
الأكاءات المتعددة وقوة الأنا لاى الطلاب

:

. $\cdot 1$

\begin{tabular}{|c|c|c|c|c|}
\hline معامل الارتباط & أبعاد قوة الأنا & م & معامل الارتباط & أبعاد قوة الأنا \\
\hline 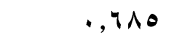 & الحكمة & 。 & .,791 & الحب \\
\hline V TAV & الأمل & 1 & 政 & الإر ادة \\
\hline סיו, & الرعاية & $v$ & 政 & الكفاءة \\
\hline - $4 \wedge q 9$ & الهلف & $\wedge$ & עتوي 1.,.,. & التفانى \\
\hline
\end{tabular}

:

-:

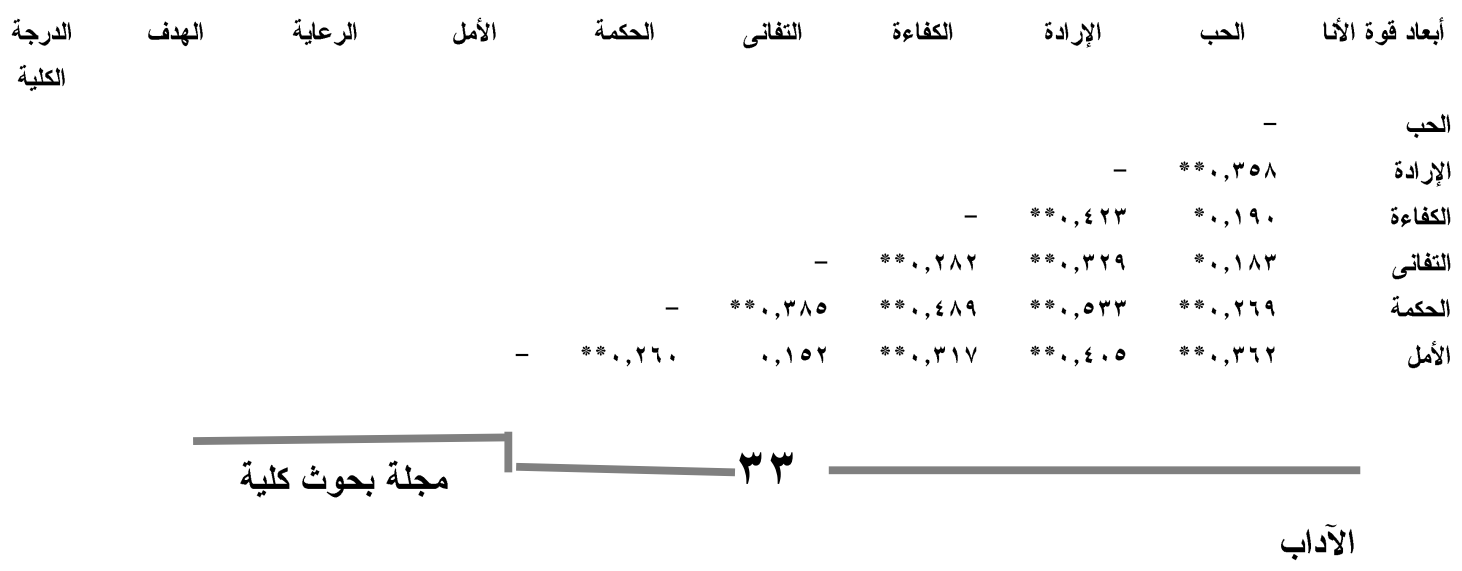


د/ سامية سمير شحاتة

\begin{tabular}{|c|c|c|c|c|c|c|c|c|}
\hline & - & $*, r, r$ & $* *, \quad, \leqslant \wedge$ & **., YrY & $*, \mid \wedge r$ & **, Yro & $* \cdot, r \leqslant V$ & الرعاية \\
\hline - & ***,YY & ***, Y & $* *, r . \varepsilon$ & 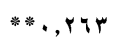 & ***,, $\leqslant$ \& & $* *$, * $\{Y V$ & $*$, * IAr & الهدف \\
\hline$* *, r, \xi \varepsilon$ & $* * *, r \leqslant \Lambda$ & ***, Y & ***, Y & $* *, r \wedge r$ & ***, ror & $* *, r \cdot r \cdot V$ & $* *, r, r \leq r$ & لـرجة الكلية \\
\hline
\end{tabular}

جدول (" ) توزيع مفردات مقياس الذكاءات المتعددة

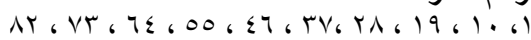

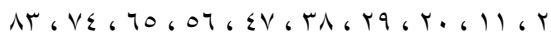

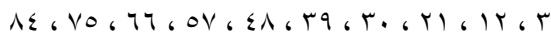

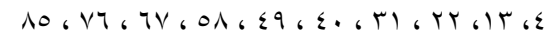

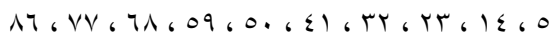

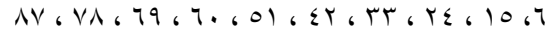

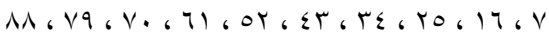

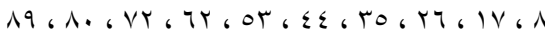

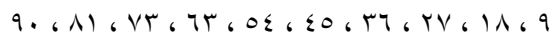

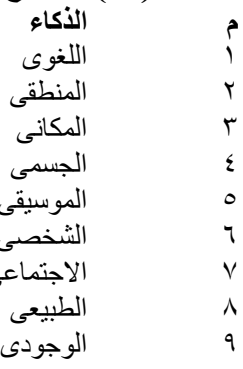


الذكاءات المتعددة وقوة الأنا لاى الطلاب

: :

جدول (11) المتوسطات والانحر افات فى أبعاد مقياس الذكاءات المتعددة وأبعاد مقياس قوة الأنا

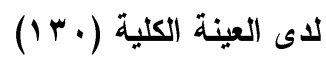

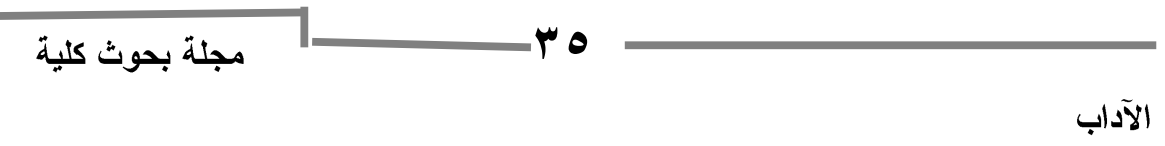




\section{د/ سامية سمير شحاتة}

\begin{tabular}{|c|c|c|c|c|c|c|c|}
\hline \multicolumn{4}{|c|}{ أبعاد قوة الأنا } & \multicolumn{4}{|c|}{ أبعاد الأكاءات المتعددة } \\
\hline الترتيب & $\varepsilon$ & م & المقاييس & الترتيب & $\varepsilon$ & م & المقاييس \\
\hline$v$ & r,^० & $r \varepsilon, V I$ & 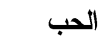 & r & $0, r y$ & $r \wedge, r_{0}$ & اللغوى \\
\hline$r$ & r,А५ & ro, $\Lambda$. & الإرادة & $\varepsilon$ & $7, .09$ & rV,Or & المنط \\
\hline - & $r, 91$ & ro,r. & الكفاءة & 1 & $r, 1$. & $r q, r r$ & 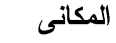 \\
\hline$\wedge$ & $r, \wedge$. & YY,OI & التفانى & $\wedge$ & $0, V_{1}$ & $r r, 9 \Lambda$ & 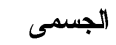 \\
\hline$\varepsilon$ & $\varepsilon, \varepsilon$ & ro, \&. & الحكمة & $r$ & $\mathrm{v}, 11$ & rA, rq & الموسيقى \\
\hline r & $r, \wedge r$ & Y , VT & الأمل & 9 & 7,94 & Yr,Y. & الثخصى \\
\hline 1 & $\varepsilon, \diamond \mathrm{V}$ & $r v, v i$ & الر عاية & 1 & $0, I V$ & YA, \& & 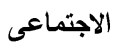 \\
\hline 1 & $\varepsilon, \diamond \mathrm{V}$ & $r \leqslant, \wedge r$ & الهـف & $\bullet$ & $\bullet, 0 \leqslant$ & rז, & الطبي \\
\hline & & & & v & $0,0 q$ & $r O, Y$ & الوجودى \\
\hline
\end{tabular}


الأكاءات المتعددة وقوة الأنا لاى الطلاب

\section{مجلة بحوث كلية}


د/ سامية سمير شحاتة 
الذكاءات المتعددة وقوة الأنا لاى الطلاب

:

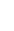

\begin{tabular}{|c|c|c|c|c|c|c|c|c|c|}
\hline الدرجة الكلية & الهاف & الرعاية & الأمل & الحكمة & التفانى & الكفاءة & الإر ادة & الحب & الأكاءوات الأنا \\
\hline., 118 &., $1 \leqslant r$ & ***, rov- &., 111 & ${ }^{* * *} \cdot, r r V$ & ., 104 &.,+11 &., 109 & - & اللغوى \\
\hline .,.१९- & ., .. & $*, r \mid \wedge$ &., .79 &.,$\cdot V \Psi$ & . I I A & $* *_{*}, r_{4},-$ & .,., ro- & ., l, r- & المنطقى \\
\hline - &., $11 \mathrm{~V}$ & $*, \mid \wedge \mu$ & ., & ***, • Y Q & $\cdot, 1 \cdot r-$ & $* \cdot, r \cdot V-$ &., $.0 v-$ & **, Yor- & المكانى \\
\hline 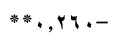 &.,.$v \mathrm{~V}$ & 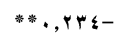 & $\because,, Y Y I-$ &.,+41 & ., $.4 \leq-$ & ., &., $1 Y \Lambda-$ & ***, & الجسمى \\
\hline$*, r Y \leqslant \varepsilon-$ &., 111 & 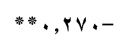 & $\cdot, \cdot v r-$ & 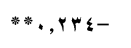 & $*, r \ldots$ & $* * *_{*} \cdot r \leqslant 0$ & $\cdot, \cdot \leq \leqslant r-$ & **a. & الموسيقى \\
\hline **a. ro. &., .11 & ***, r०१- & $\because,, \mid \vee \Psi-$ & .,.ar & .,.. q- & $*,|,| \vee \mu-$ &., $.9 v-$ & *** & الثخصى \\
\hline.,., $\mathrm{V}$ &., $1 \%$. & E &.,+11 & $\cdot, \ldots \circ-$ & .,.or- & ${ }^{* * *} \cdot$, , YVA- & .,. ro & ,.,rr- & الاجتماعى \\
\hline , , $\leqslant$ V &., $11 \mathrm{v}$ & $\cdot, 1 \cdot v-$ & ${ }^{*}{ }^{*} \cdot, r r \Lambda-$ & $\cdot,+11$ &., $1 \leqslant \psi$ &., $10 .-$ & . & ., & الطبيعى \\
\hline .,. or- & **, RTA & **: &., .94 & . & ד & .,.rr- & .,.rq & ., IYV- & الموجودى \\
\hline.,.$\vee$. & .,Ir| & $*_{*}^{*}, r Y Y$ & ., lro- & $\begin{array}{r}., 1.9- \\
., Y r\end{array}$ & $\begin{array}{l}* *,+r 19 \\
=\ldots+1\end{array}$ & 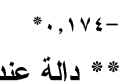 & $\begin{array}{l}., \nabla v \varepsilon \\
., 1 \vee \mu\end{array}$ & دالة ه|,.. & الدرجة الكلية \\
\hline
\end{tabular}


د/ سامية سمير شحاتة

..p

( labuvie,1990). 
الأكاءات المتعددة وقوة الأنا لاى الطلاب

.

مجلة بحوث كلية 
د/ سامية سمير شحاتة

., 0

. , , 
الأكاءات المتعددة وقوة الأنا لاى الطلاب

\section{مجلة بحوث كلية}


د/ سامية سمير شحاتة

.$\left(\varepsilon V-\varepsilon 0: Y_{N} V_{\text {。 }}\right.$

$\cdot \cdot 9$

R.Trees et al. ,2010)

$\cdot \cdot, 1$ 
الأكاءات المتعددة وقوة الأنا لاى الطلاب

$\cdot \cdot \cdot 1$

$\cdots, 0$

مجلة بحوث كلية

$\varepsilon 0$

الآداب 
د/ سامية سمير شحاتة

."

: 
الأكاءات المتعددة وقوة الأنا لدى الطلاب

\begin{tabular}{|c|c|c|c|c|c|c|}
\hline \multirow[t]{2}{*}{ الدلالة } & \multirow[t]{2}{*}{ ت } & \multicolumn{2}{|c|}{ الإحاث ن= 1 } & \multicolumn{2}{|c|}{ الأكور ن=ء } & \multirow[t]{2}{*}{ العينة } \\
\hline & & $\varepsilon$ & م & $\varepsilon$ & 5 & \\
\hline غ.دال & $\cdot, 909$ & $0,1 \leqslant 7$ & $r v, q . q$ & $0,0 \wedge \wedge$ & YA,AIr & اللغوى \\
\hline غ.دال &.,$Y 41$ & $0, r V$ & $r v, r q$ & $7, \wedge 1$ & rV,TVI & المنطقى \\
\hline غ.دال & ., VMr & $7, \leqslant 99$ & $r q, \ldots$ & $r, \cdot v$ & Y7,707 & المكانى \\
\hline., .1 & $r, r \leqslant \wedge-$ & $0, v_{0}$ & $r \otimes, \Delta V$ & $0, r r$ & $r Y, r \varepsilon$ & الجسمى \\
\hline ع.دال & $\cdot, \Lambda \cdot 7-$ & $\mathrm{V}, . \mathrm{T}$ & 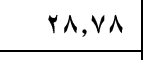 & $\mathrm{V}, 1 \wedge$ & $r v, V \wedge$ & الموسيقى \\
\hline$\cdot, .0$ & $r, r \ldots-$ & $\neg, \wedge \vee$ & rr,or & $\checkmark, V \leqslant$ & $r \cdot, \Lambda I$ & الشخصى \\
\hline ع.دال & .,AYV & $7, \varepsilon$. & 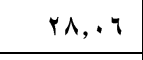 & $r, \leqslant q$ & $Y \wedge, \wedge 1$ & الاجتماعى \\
\hline غ.دال & $1, \wedge Y \varepsilon$ & 0,91 & Yo,VV & $0, \cdot r$ & rV,Or & الطبيعى \\
\hline غ.دال & 1,orq- & 7,00 & ro,qr & $\varepsilon, r$. & $r \leqslant, \varepsilon r$ & الوجودى \\
\hline غ.دال & $1,1 \times 0$ & $\{1, \wedge r$ & $r \cdot\{, \leqslant Y$ & ro,qr & YI,TV & المجموع الكلى \\
\hline$\ldots 0$ & $r, r \otimes \wedge-$ & $\varepsilon, \pi$ & ro,Or & $1, \varepsilon r$ & $r \leqslant, 1.9$ & الهـف \\
\hline غ.دال & $\cdot, \cdot \wedge 1$ & $r, 91$ & $r q, V \leq$ & $r, v \cdot \varepsilon$ & rq,Vq & الأمل \\
\hline ع.دال & . , YrA & $\varepsilon, 1.9$ & rV,Tr & $0, \cdot r$ & $r v, \wedge 1$ & الرعاية \\
\hline$\ldots 0$ & $r, \cdot I r-$ & $\varepsilon, \diamond V$ & rq,17 & $\varepsilon, r \varepsilon$ & $r \varepsilon, Y$. & الحكمة \\
\hline$\cdots+1$ & Y,VTV- & r,TV & $r \otimes, \wedge v$ & $1,0 \mathrm{~V}$ & $r \varepsilon, 0$. & الكفاءة \\
\hline غ.دال & $\cdot, \cdot \varepsilon V$ & $\varepsilon, T Y$ & Yr,O. & r, Vo & YY,Or & التفانى \\
\hline غ.دال & $\cdot, \nabla \wedge r$ & $r, v r$ & $r \leqslant, \varepsilon 0$ & $r, 9 q$ & $r \leqslant, 9 \wedge$ & الحب \\
\hline غ.دال & $\cdot, \wedge 9 \mathrm{r}-$ & $\varepsilon, 711$ & $r 7,1.7$ & $r, q \ldots$ & Yo,0. & الإر ادة \\
\hline غ.دال & $1, \wedge 0$. & $19,7.7$ & $r \cdot r, \varepsilon \cdot q$ & $1 \Lambda, r v$ & $r \cdot 9,0 V$ & المجموع الكلى \\
\hline
\end{tabular}


د/ سامية سمير شحاتة

- 
الأكاءات المتعددة وقوة الأنا لاى الطلاب

\section{مجلة بحوث كلية}

$\varepsilon q$

الآداب 
د/ سامية سمير شحاتة

0. 


$$
\begin{aligned}
& \text { إبر اهيم عيد. أزمات الثباب النفسية، القاهرة ـ مكتبة زهراء الثرق.209 - ب ـ ـ . }
\end{aligned}
$$

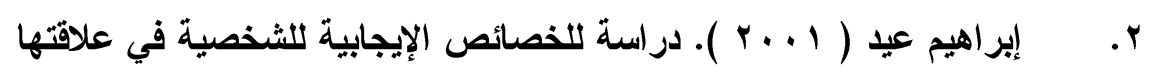

$$
\begin{aligned}
& \text { بمتغيري النوع }
\end{aligned}
$$

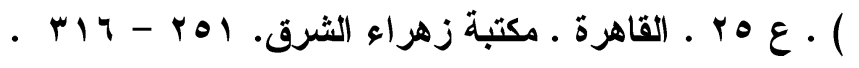

$$
\begin{aligned}
& \text { ـ الجامعة الإسكندرية ـ دار المعرفة الجامعية. ط؛. } \\
& \text { ـ مؤسسة عز الدين للطباعة و النشر. }
\end{aligned}
$$

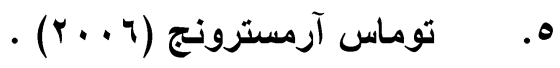

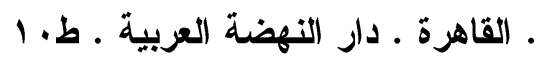

$$
\begin{aligned}
& \text { • القاهرة ـ دار الفكر العربى • طا }
\end{aligned}
$$

• ترجمة جابر عبد الحميد جابر .القاهرة ـ دار النهضة العربية. • ترجمة عبد الله عريف. بنغازي • جامعة قاريونس.

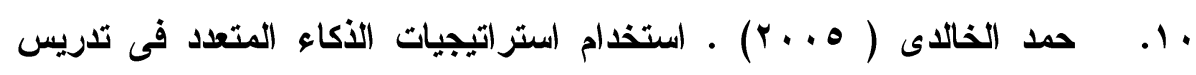

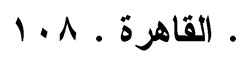

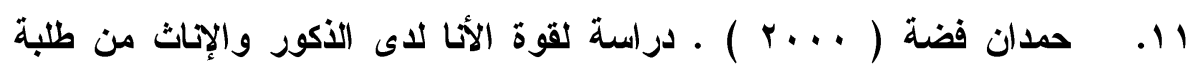
الجامعة قاطني القرى و المدن وعلاقتها بالمستوى الاجتماعي الثقافي والاقتصادي لايهم • ع ع r المجلس الوطني للثقافة والفنون والآداب، الكويت.

$$
\text { مجلة بحوث كلية }
$$


ـ القاهرة ـ مؤسسة مختار للطباعة والنشر.

ـ كلية التربية ـ جامعة الأزهر.

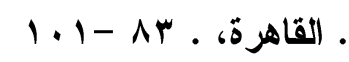

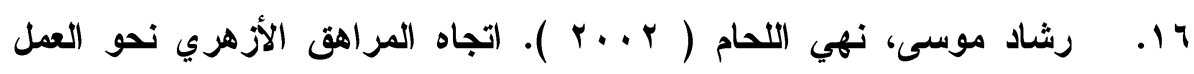

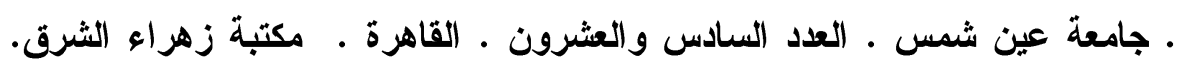

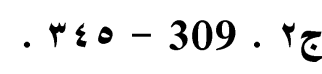

• رسالة ماجستير غير منشورة ـ الرياض . جامعة الملك سعود ـ ـلية التربية .

ـ الكتاب الأول ـ ترجمة مدارس الظهران الأهلية ـ المملكة العربية السعودية.

القاهرة : مكتبة وهبه.طس .

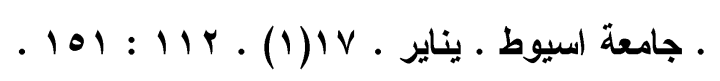

• م القاهرة ـ الأنجلو المصرية.

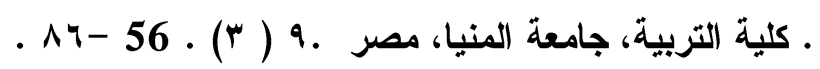

ـ الجمعية المصرية للار اسات النفسية ـ القاهرة ـ مكتبة الأجلو المصرية ـ 11(Y) .

• الاردن مكتبة الفرقان . 


\section{الأكاعات المتعددة وقوة الأنا لدى الطلاب}

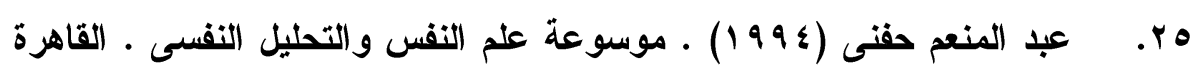

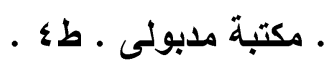

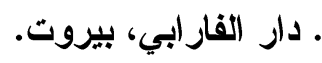

ف فلسطين . آفاق.طا

• القاهرة ـ دار قباء للطباعة و النشر و التوزيع .

ـ القاهرة ـ الأنجلو المصرية . . 1 - . . .

• رسالة ماجستير غير منشورة ـ كلية التربية. الجامعة الإسلامية. غزة.

• مجلة كلية التربية ، جامعة الزقازيق العدد (هـ) ـ يناير •

1ط. عمان . الاردن . دار الفكر .

ـ الكويت ـ دار سعاد الصباح.طا

ـ ترجمة سامي على وعبد السلام القفاث ـ القاهرة .دار المعارف.

• رسالة ماجستير غير منشورة . كلية التربية . جامعة عين شس.

.1 b.

مجلة بحوث كلية

or 
45. Al-Balhan , E.(2006). Multiple Intelligence Styles in Relation to Improved Academic Performance in Kuwaiti Middle School Reading . Digest of Middle East Studies . 15 ( 1 ). 18-34.

46. Barnes, B. \& Shrinivas, R. (1993) . Personality traits of selfactualized

women. Psychological Studies, 38 (1).7-9.

47. Campbell, Linda; Campbell,Bruce \& Dickinson, Dee, (1999) . Teaching \& Learning Through Multiple Intelligences. Second Edition, Allyn\& Bacon, U.S.A, Internet: www.abacon.Com.

48. Chan , D. (2003). Multiple Intelligences and Perceived Self Efficacy Among Chinese Secondary School Teachers in Hong Kong . Educational Psychology, Vol. 23 ( 5 ). 521-533.

49. Checkley, Kathy (1997) .The first seven and the eighth: a conversation with Howard Gardner. Educational Leadership , V.55, No. BEDI 97022212.8:13. 
الأكاءات المتعددة وقوة الأنا لاى الطلاب

50. Cutshall , L.(2003). The Effects of Student Multiple Intelligence preference on Integration of Earth Science Concepts and Knowledge within a Middle Grades Science Classroom . Master of Arts , Johnson Bible College. .1-47.

51. Deri, S. (1990): Changing Concepts of the Ego in psychoanalytic

theory, psychoanalytic Review, vol. 77(4) . 511-518.

52. Erikson, E. H. (1968). Identity: Youth and crisis. New York: Norton.

53. Erikson, E. H. (1985). The life cycle completed. New York: Norton

54. Erikson, E.H. (1964). Insight and responsibility. New York: Norton

55. Erikson, E.H. (1963a). Childhood and society. (2nd. ed.). New York: Norton.

56. Fontan‘D.،(1995). Psychology for teachers.3ed.Macmillan press، LTD.

57. Furnham, A. \& Akande, A. (2004). African Parents Estimates of Their Own and Their Children's Multiple Intelligences . Current Psychology : Developmental, Learning, Personality, Social . 22 (. 4 ) .281-294.

58. Gardner, H. \& Hatch ,T.(1989). Multiple intelligences go to school . Educational Researcher .18(8) ..4-10.

59. Gohlinghorst , N. \&Wessels , B. (2001). Enhancing Student Achievement in Social Studies through the Use of Multiple Intelligences. Master of Arts Action Research Project, Field Based Master's Program Saint Xavier University and IRI / Skylight . Dissertations, .1-80.

60. Harry , M. (1992). An Analysis of Gardner's Theory of Multiple Intelligence . Paper presented at the Annual Meeting of the Eastern. Educational Research Association ,.1-40.

61. Jody Koening; April R.Trees ; Paul Schrodt and et al (2010 ). Exploring Links Between Well-Being and Interactional Sense-Making in Married Couples' Jointly Told Stories of Stress . Journal of Family Communication . 10(3) .174-193 .

62. Loori , A. (2005). Multiple Intelligences : A comparative Study Between The Preferences Of Males and Females . Social Behavior and Personality . 33 ( 1 ) .77-88.

Markstrom, C\& Marshall; S.K. .. ( $r . . y)$. The Psychosocial Inventory of ego strengths. . Examination of theory and psychometric properties - Journal of Adolescence .February .30 (1), pg. 63-79

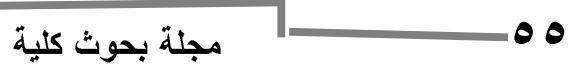




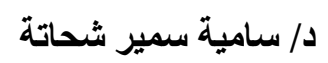

63. Markstrom, C., Sabino, V., Turner, B., \& Berman, R. (1997). The Psychosocial Inventory of Ego Strengths: Development and validation of a new Eriksonian measure. Journal of Youth and Adolescence, 26. 705-732.

64. Markstrom. C. A., and Kalmanir, H. (2001). Linkages between the psychosocial stages of identity and intimacy and the ego strengths of fidelity and love. Identity. International Journal of Theory and Research, 1( 2). 179-196.

65. Murphy \& David Shefer (1994) .

66. Nolen , J . (2003). Multiple Intelligences In The Classroom . Education . 124(1 ) . 115-119.

67. Rogalla , M. \& Margison , J. (2004) . Future Problem Solving Program Coaches Efficacy in Teaching For Successful Intelligence and Their Patterns Of Successful Behavior . Reaper Review .26 (.3 ). 175-177.

68. Seghers, Myles M. \& Baker, Jane O.(2005). Multiple Intelligence Theory: Cognitive Development Young Adolescents: http://www. Tec. Nsula.Edu/ LMSA/MI Theory . htm

69. Shearer , B. (2004). Using Multiple Intelligences Assessment to Promote Teacher Development and Student Achievement. Teacher College Record , .106(1) .147-162

70. Sinha, V. \& Prabhat, R. (1993): Relationship between egostrength and job satisfaction, Indian Journal of Psychometry and Education,24 (2), 99 -102.

71. Snyder, C. R., Hoza, B., Rapoff, W. E., Ware, L., Danovsky, M., Highberger, L., Rubinstein, H., \& Stahl, K. J. (1997). The development and validation of the children's hope scale. Journal of Pediatric Psychology, 22, 399- 421.

72. Snyder, M., Clary, E. G., \& Stukas, A. A. (2000). The functional approach to volunteerism. In G. R. Maio \& J. M. Olson (Eds.), Why we evaluate: Functions of attitudes (pp. 365-393). Mahwah, NJ: Erlbaum.

73. Teele, Sue ,(1999). Rainbows Of Intelligence, Exploring How Students Learn. Sue Teele \& Associates, U.S., Redlands CA. 74. Wolman‘B.B(1989). Dictionary of behavioral science. Academic Press، Inc.، san Digo.

Multiple Intelligence and with Ego Strength of the students Dr. samia samir shehata 
The research aims to study the multiple intelligences and the Ego Strength of students in King Saud University. Sample was selected consisting 130 student and a student of university students. (66) males and (64) females. Average age (25.18) and a standard deviation (1.56). Scale was applied to the social psychology of ego-strength Invontry of Maxtröm et al. and The measure of multiple intelligences of McKinsey. The results showed that the intelligences enjoyed by members of the sample according to their evaluations of themselves in descending order were as follows: social intelligence (Inter-personal), linguistic intelligence, musical intelligence, logical intelligence, natural intelligence, spatial intelligence, existential intelligence, bodily intelligence profile. The results also showed that the dimensions of ego strength enjoyed by members of the study sample according to their evaluations of themselves in descending order were as follows: care, hope, will, wisdom, cometence, purpose, love ,fidelity. Results also showed a correlation function between the dimensions of multiple intelligences and the strength of the ego as follows: the existence of correlation function between linguistic intelligence and both the wisdom and care; and logical intelligence, care and will; a correlation between visual spatial intelligence and both the care and love and will; a correlation between physically intelligence, both of love and care, hope and the total degree of the strength of the ego; a correlation between music intelligence, both of cometence and, fidelity, love and wisdom, care, and the total degree of the strength of the ego; a correlation between interpersonal intelligence and cometence; a correlation between intrapersonal intelligence and both love and care, competence and hope and the total degree of the strength of the ego; a correlation between natural intelligence and hope; a correlation between existential intelligence and both proupse and care; and finally the existence of correlation between the total score of multiple intelligence and both of fidelity and care and cometence. The results also showed that there are no differences between males and females in both the linguistic and logical , spatial, music, social and natural intelligence and the total score of multiple intelligence In the hope and care and fidelity and love and the will dimensions and the total degree of the strength of the ego. While others have found significant differences between males and females in physical and personal intelligence and the puropse, wisdom and cometence dimensions of the strength of the ego.

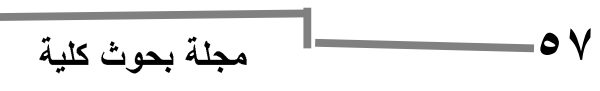

